$\mathrm{E}$

EVALUAR
2017, Vol. 17, No 2

ISSN 1667-4545

Recuperado de https://revistas.unc.edu.ar/index.php/revaluar

Laboratorio de Evaluación Psicológica y Educativa

Facultad de Psicología - Universidad Nacional de Córdoba

\title{
Inhibición de Borrado en Adultos Mayores: Aportes para la Validación de una Tarea
}

\author{
Deletion Inhibition in Older Adults: \\ Contributions for the Validation of a Task
}

\author{
Ana Comesaña ${ }^{*}$, Florencia Stelzer ${ }^{1}$, Isabel Introzzi ${ }^{1}$ \\ 1- Instituto de Psicología Básica, Aplicada y Tecnología (IPSIBAT) - Consejo Nacional de Investigaciones \\ Cientificas y Técnicas (CONICET)- Universidad Nacional de Mar del Plata (UNMdP), Argentina.
}

Recibido: 09/08/2017 Revisado: 06/10/2017 Aceptado: 13/10/2017

Introducción

Materiales y

métodos

Procedimiento

Resultados

Discusión

Referencias

\section{Resumen}

La inhibición de borrado constituye un tipo inhibitorio que permite eliminar la información irrelevante durante la ejecución de una tarea en curso. Los instrumentos o pruebas para evaluarla son escasos. El objetivo de este trabajo es obtener evidencia de validez de constructo utilizando una tarea informatizada de la TAC (Tareas de Autorregulación Cognitiva) para medir inhibición de borrado. Para este estudio, se utilizó una muestra de 53 participantes (32 adultos jóvenes y 21 adultos mayores). Los análisis realizados indicaron que la tarea cumple con los supuestos de estructura interna de la tarea y los cambios maduracionales esperados para este proceso. La tarea resulta un instrumento apropiado para la medición de la inhibición de borrado en sujetos adultos jóvenes y mayores.

Palabras clave: funciones ejecutivas, inhibición de borrado, tarea informatizada, adultos mayores, validación de constructo

\begin{abstract}
Deletion inhibition constitutes an inhibitory kind which allows for the elimination of irrelevant information during the execution of a task. The instruments or tests for evaluating it are scarce. The aim of this work is to obtain evidence of construct validity using a computerized task from the TAC (cognitive auto-regulating task) to measure deletion inhibition. Fifty three adults (32 young adults and 21 older adults) participated in this study. The analyses carried out indicate that the task fulfills the assumptions of internal structure and the maturational changes expected for this process. The task is an appropriate instrument for measuring deletion inhibition in young and older adults.
\end{abstract}

Keywords: executive functions, deletion inhibition, computerized task, older adults, construct validity

\footnotetext{
*Correspondencia a: Dra. Ana Comesaña a: Matheu 3074 (7600). 549223 560-9511 Mar del Plata, Argentina. acomesan@mdp.edu.ar Cómo citar este artículo: Comesaña, A., Stelzer, F., \& Introzzi, I. (2017). Inhibición de borrado en adultos mayores: Aportes para la validación de una tarea. Revista Evaluar, 17(2), 143-153. Recuperado de https://revistas.unc.edu.ar/index.php/revaluar

Agradecimientos: Se agradece la colaboración de todos los participantes de esta investigación. Este trabajo se enmarcó en un proyecto financiado por la Secretaría de Ciencia y Tecnología y Coordinación de la Universidad Nacional de Mar del Plata ("Desarrollo de la regulación de la cognición, el comportamiento y la emoción durante el ciclo vital”) y fue desarrollado durante los años 2015-2016. También se debe agradecer al CONICET, institución a la cual pertenecen las autoras como investigadoras.

Las autoras manifiestan no tener conflicto de intereses.
} 


\section{Introducción}

La inhibición contribuye de manera significativa a nuestra vida cotidiana, a la autorregulación y a la adaptación al medio ambiente a través del control de nuestros pensamientos, comportamientos y emociones, nos permite adaptarnos. Si bien no existe una única definición de este proceso, la mayoría de los modelos actuales concuerdan con que su función principal consiste en limitar el ingreso de información irrelevante a la conciencia o foco atencional, lo que permite mantener activa la información relevante para el logro de objetivos (Diamond, 2013; Introzzi, Canet-Juric, Aydmune, \& Stelzer, 2016). Por ejemplo, luego de una fuerte discusión es probable que los recuerdos y pensamientos vinculados con ella se presenten de manera intrusiva en nuestra conciencia, compitiendo por los recursos atencionales e interfiriendo con la tarea que estemos realizando (p. ej., una actividad laboral). En esta situación, resulta esencial la habilidad para suprimir o eliminar del foco atencional tales representaciones, dado que estas afectan negativamente nuestro desempeño.

Aunque actualmente existe un acuerdo sobre la importancia de la inhibición para la adaptación y el adecuado funcionamiento de otras funciones cognitivas, se observan divergencias en cuanto a su definición conceptual y operativa. Se distinguen dos enfoques principales: uno que entiende a la inhibición como una función global y unitaria (Cohen, Dunbar, \& McClelland, 1990; Morton \& Munakata, 2002) y otro que fragmenta la inhibición en un conjunto de procesos con características operativas distintivas: la inhibición de acceso, que se ocupa de controlar el acceso de la información al foco atencional, la inhibición de borrado, que tiene como objetivo borrar del foco atencional y de la memoria de trabajo la información irrelevante para una tarea en curso $\mathrm{y}$, finalmente, la inhibición de restricción, que se encarga de suprimir o restringir comportamientos prepotentes pero inadecuados para los objetivos actuales. De todas ellas, la inhibición de borrado, también llamada cognitiva, es la que ha recibido la menor atención.

Dentro de la línea de los enfoques fragmentados o multidimensionales (Miyake \& Friedman, 2012), uno de los más representativos es la "Teoría o Hipótesis de la Ineficiencia Inhibitoria" propuesta por Hasher y Zacks (1988) y revisada en distintas oportunidades (Hasher, Zacks, \& May, 1999; Healey, Ngo, \& Hasher, 2014; Lustig, Hasher, \& Zacks, 2008). Según esta teoría, la causa subyacente de la disminución en el funcionamiento cognitivo en los adultos mayores es un déficit inhibitorio. Algunos autores sostienen que la afectación en los adultos mayores varía entre los distintos subtipos de inhibición (Vadaga, Blair, \& Li, 2015). En relación con la inhibición de borrado durante el envejecimiento, la disminución de su eficiencia se manifiesta en una mayor dificultad para borrar la información irrelevante del foco atencional o para disminuir su activación desde la memoria de largo plazo en comparación con otros grupos etarios (Ortega, Gómez-Arizaga, Román, \& Bajo, 2012). Es decir que la información que previamente resultó relevante para el logro de algún objetivo o alguna tarea actualmente tiene que ser olvidada o borrada.

La relevancia de la inhibición para explicar el declive cognitivo asociado con el envejecimiento impulsó el desarrollo de paradigmas destinados a su evaluación. Uno de los instrumentos de evaluación más utilizados es la prueba diseñada según el paradigma del olvido dirigido con reconocimiento propuesta por Oberauer (2001; 2005) que es una adaptación del paradigma original de Stenberg (1969) retomada por otros autores (Aslan \& Baüml, 2012; Sahakyan, Delany, \& Goodmon, 2008; Sego, Goldin, \& Gottlob, 
2006). Para este tipo de pruebas los sujetos deben memorizar dos listas de palabras, luego aparece una señal de olvido que indica cuál de las listas deben retener en su memoria y finalmente se efectúa una prueba de reconocimiento. En la prueba de reconocimiento los sujetos deben identificar la pertenencia o no de una serie de ítems de prueba a los de una lista que debían retener en la memoria. Los ítems de prueba pueden ser relevantes (PR; pertenecían a la lista de estímulos que debían recordar); irrelevantes (PI; pertenecían a la lista de estímulos que debían olvidar) o nuevos (PN; no se hallaban en ninguna de las dos listas). Distintos estudios con este paradigma indicaron mayores tiempos medios de respuesta (TR) para los PI en comparación con los PN. Asimismo, reportaron un menor porcentaje de respuestas correctas frente a los PI respecto de los PN (precisión; Joorman \& Gotlib, 2008; Oberauer, 2001). Los PI constituyen información que se torna irrelevante y debe borrarse de la memoria, por lo que las diferencias observadas en los TR y la precisión entre PI y PN se definen como índices para medir la eficiencia operativa de la inhibición de borrado.

Si bien el paradigma desarrollado por Oberauer (2001) constituye un instrumento válido de medición de la capacidad de inhibición de borrado, su utilización en participantes de distinta cultura exige adaptaciones, dado que los estímulos de la prueba original eran verbales. Diferentes estudios han señalado que la extensión fonológica, la familiaridad y frecuencia de uso y la valencia emocional afectan la retención de los conceptos y el nivel de interferencia en la memoria de trabajo (Baddeley, 2012; Cowan et al., 2005). Además, la familiaridad y la frecuencia de uso de ciertas palabras varían para diferentes grupos etarios y ambientes socioculturales (Vivas, Vivas, Comesaña, García-Coni, \& Vorano, 2016; Yao, Wu, Zhang, \& Wang, 2016). Según nuestros conocimientos, en Latinoamérica no se cuenta con instrumentos válidos y confiables para la medición de la inhibición de borrado que puedan ser utilizadas en diferentes grupos de edad, y adaptables en diferentes contextos socioculturales. Se optó por diseñar un instrumento con estímulos visuales (figuras abstractas no codificables semánticamente), que permita controlar el efecto de las variables psicolingüísticas en el procesamiento de los estímulos.

Por todo lo anterior, el principal objetivo de este trabajo consiste en presentar evidencia preliminar sobre la validez de constructo de una prueba informatizada diseñada para medir la inhibición de borrado. Esta prueba se enmarca en una batería informatizada (Tareas de Autorregulación Cognitiva, TAC) desarrollada por Introzzi y Canet Juric (Introzzi, Andrés, Canet-Juric, \& Richard's, 2016) y diseñada especialmente para evaluar distintos procesos cognitivos. Como explica Hogan (2015), los estudios de validación resultan de especial interés ya que proporcionan evidencia empírica sobre el grado en que las puntuaciones obtenidas representan indicios adecuados del rasgo o constructo de interés. En este trabajo se presentan dos tipos de evidencias de validez: la validez de estructura interna y la validez de cambios maduracionales o del desarrollo (ambas contribuyen a la validez del constructo) utilizando la prueba de inhibición de borrado en cuestión siguiendo los criterios mencionados por Hogan (2015).

\section{Materiales y métodos \\ Participantes}

La muestra inicial fue seleccionada de manera intencional no probabilística y dividida en dos grupos etarios: uno integrado por adultos jóvenes $(\mathrm{n}=35)$ de 20 a 40 años y el otro por adultos mayores de más 65 años $(n=34)$, de la ciudad de Mar del Plata, Argentina.

Los criterios de inclusión para ambos gru- 
pos fueron: ausencia de diagnóstico de enfermedades psiquiátricas y/o neurológicas, focales o degenerativas (depresión mayor, esquizofrenia, ACV, DCL, esclerosis múltiple, Alzheimer, Parkinson, entre otras) y un nivel de educación formal superior a los siete años. Además, para el grupo de adultos mayores fue un requisito obtener 82 puntos o más en el ACE- R (Adenbrook`s Cognitive Examination Revisado; Torralva et al., 2011), ya que este es el puntaje de corte señalado en esta adaptación para sospechar deterioro cognitivo; por esta razón fueron excluidas de este grupo ocho personas. También se excluyeron de la muestra final cinco adultos mayores y tres jóvenes que se alejaban más de tres desvíos típicos del rendimiento promedio de sus respectivos grupos.

La muestra final quedó conformada por 32 adultos jóvenes con una $\mathrm{M}_{\text {edad }}=28.75, \mathrm{DE}=6.08$ (65.6\% mujeres y $34.4 \%$ varones) y por 21 adultos mayores $\mathrm{M}_{\text {edad }}=69.43 \mathrm{DE}=5.73(66.7 \% \mathrm{mu}-$ jeres y $33.3 \%$ varones).

\section{Instrumentos}

$\boldsymbol{A C E}$ - R. Dada la alta prevalencia de los trastornos cognitivos en los adultos mayores de 60 años, se administró el ACE-R como como método de screening para discriminar entre el envejecimiento normal y el patológico y utilizar a este último como criterio de exclusión. El ACE (Addenbrooke's Cognitive Examination; Mathuranath, Nestor, Berrios, Rakowicz, \& Hodges, 2000) es un test breve que evalúa distintos dominios cognitivos para diferenciar entre envejecimiento normal y patológico y entre demencia tipo Alzheimer y demencia Frontotemporal. El puntaje máximo es de 100 y evalúa: orientación (10), atención (8), memoria (35), fluencia verbal (14), lenguaje (28) y habilidades visuoespaciales (5). La adaptación del ACE-R en español (Torralva et al., 2011) mostró un nivel de confiabilidad interna alta (alfa de Cronbach $=.89)$. La validez concurrente, determinada por la correlación entre el ACE-R y el CDR (Clinical Dementia Rating; Hughes, Berg, Danzinger, Coben, \& Martin, 1982), fue estadísticamente significativa $(\mathrm{p}<.001)$ y la concordancia entre evaluadores fue alta (kappa de Cohen $=.98)$. Prueba de inhibición de borrado visual (TAC). Esta prueba se diseñó con base en el paradigma de Oberauer (2001) de olvido dirigido y reconocimiento. La prueba original, al igual que la presentada aquí, está estructurada en tres fases: En la fase de aprendizaje (fase 1) se deben memorizar dos listas de estímulos (figuras abstractas en rojo o azul) distribuidos en filas de uno a tres elementos localizados unos en la mitad superior de la pantalla y otros en la mitad inferior, los de la mitad superior se presentan siempre en color rojo y los de la mitad inferior en azul. En la fase señal (fase 2): se le indica al participante sobre qué lista realizará la fase de reconocimiento (lista target). $\mathrm{Si}$ la señal consiste en un rectángulo azul, en la fase de reconocimiento se evaluará el recuerdo de la lista azul y si la señal es un rectángulo rojo, se evaluará el recuerdo de la lista roja. El 50\% de los ensayos evalúa la lista azul y el otro $50 \%$ la roja y su distribución es aleatoria a lo largo de toda la prueba. Por último, en la fase de reconocimiento (fase 3), el rectángulo se mantiene en la pantalla $\mathrm{y}$ en el centro aparece una figura en color blanco -ítem de prueba- y los participantes deben decidir si forma parte o no de la lista target con la mayor rapidez y precisión posible presionando la tecla "Z" ( si considera que estaba en la lista target) y la tecla "M" (si no estaba en esa lista). Se distinguen tres tipos de ítems de prueba: el 50\% son ítems o probes relevantes o pertenecientes a la lista target (PR), el 25\% son ítems o probes intrusos o pertenecientes a la lista irrelevante o por ser borrada (PI) y el 25\% restante son ítems o probes nuevos (PN) o no contenidos en ninguna de las dos listas. 
Estructura de los ensayos. Cada ensayo comienza con un punto de fijación que permanece en pantalla durante 200 milisegundos (ms); luego se presentan las dos listas con las figuras abstractas (ubicadas en la parte superior e inferior de la pantalla). Por cada figura se computan $1300 \mathrm{~ms}$, por lo que el tiempo de visualización es el producto de la multiplicación del número total de figuras por $1300 \mathrm{~ms}$. Luego de un intervalo de $800 \mathrm{~ms}$, aparece un rectángulo (azul o rojo) en el centro de la pantalla durante $200 \mathrm{~ms}$. Por último, el tiempo que separa la señal del ítem de prueba, que aparece en color blanco, es de $300 \mathrm{~ms}$ (ver Fig.1). Los principales índices de desempeño de esta prueba son: (a) las diferencias entre el tiempo medio de respuesta (calculado solamente para las respuestas correctas) en el reconocimiento de los PI y el tiempo medio de respuesta en el reconocimiento de los PN (TR medio PI - TR medio PN); (b) la diferencia en el porcentaje de respuestas correctas entre el reconocimiento de los PN y de los PI (respuestas correctas PN - respuestas correctas PI). Cuanto mayor es la diferencia en a y $b$, menor es la eficiencia del funcionamiento de la inhibición de borrado puesto que para obtener un buen desempeño, el participante debe ser capaz de eliminar de su memoria de trabajo los PI que corresponden a la lista irrelevante tras la señalización.

Cambios sobre la versión original. A la versión original de la prueba se le realizaron algunos cambios para adaptarla a nuestro medio: (a) se reemplazaron los estímulos verbales por estímulos visuales -figuras abstractas- a fin de dificultar el uso de estrategias de procesamiento verbal de los estímulos y (b) se redujo la cantidad de ensayos que integraban la prueba original con el propósito de disminuir el probable efecto de fatiga debido a la extensión (eran 64 ensayos precedidos por 12 de práctica y en la versión actual son 32 ensayos precedidos por 5 de práctica). Los estímulos no verbales fueron seleccionados de entre un total de 200 figuras abstractas diseñadas especialmente para esta prueba. Estas fueron sometidas a una evaluación de 20 participantes a los que se les solicitó que nombraran la imagen que veían en cada una de ellas. Fueron eliminadas aquellas figuras en las que coincidieron en su denominación dos o más participantes. Finalmente, quedaron 100 figuras que fueron utilizadas como estímulos para el diseño de los 32 ensayos de la tarea. Cada ensayo presenta tres fases sucesivas: aprendizaje, señal y reconocimiento (ya mencionadas en el apartado anterior).

Se muestran ejemplos de los tipos de ítems de prueba y las tres fases de la tarea para medir la inhibición de borrado de la batería informatizada TAC (Introzzi, Andrés, et al., 2016) junto con los tiempos de presentación en la Fig.1 a continuación.

(a)
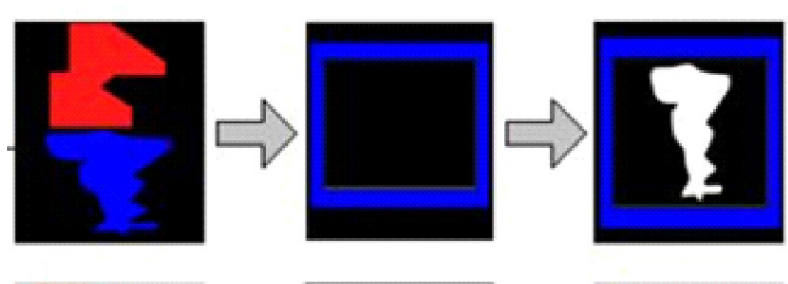

(b)
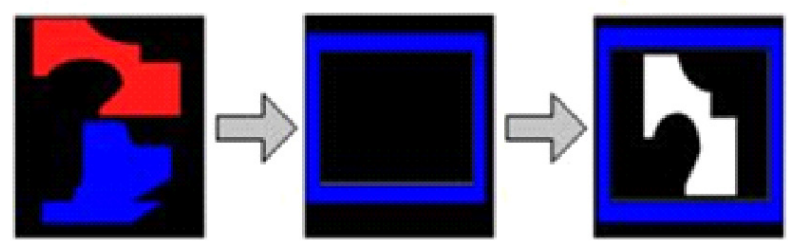

(c)
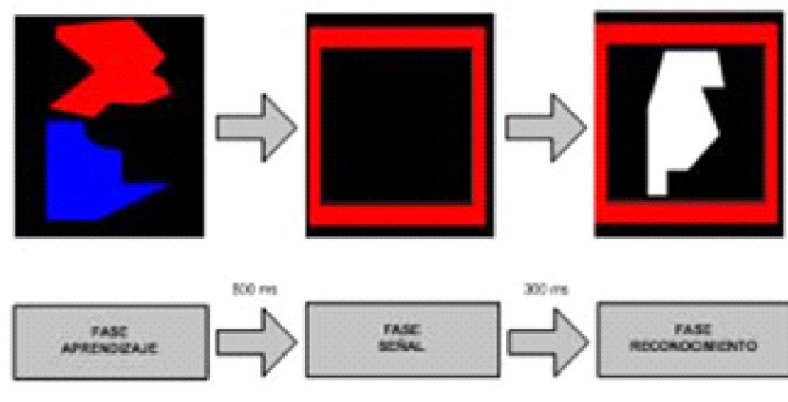

mecomonento

EECONDONANTO

Figura 1

Ejemplo de secuencia de tres ensayos con listas de $1 \mathrm{x} 1$ estímulos de la tarea TAC.

Nota. Ejemplos de ensayos con distintos ítems de prueba (a) Relevante, (b) Irrelevante y (c) Nuevo,y los tres tipos de fases de la tarea. 


\section{Procedimiento}

La participación fue voluntaria. Primero se les presentó a los participantes el consentimiento informado que explicaba los objetivos, las pruebas a ser administradas en esta investigación, el tratamiento y el uso confidencial de los datos conforme con la Declaración de Helsinki (Asociación Médica Mundial, 2013), y en consonancia con los principios éticos y el código de conducta de los psicólogos establecido y reformulado por la APA (2017). También se siguieron los procedimientos indicados por la Ley Nacional Argentina No25.326 (2000) de protección de datos personales reglamentada por el decreto 1158/2001. La administración se realizó en una entrevista individual con una duración de entre 20 a 30 minutos. Se aplicó la prueba de borrado visual de la TAC (Introzzi, Andrés, et al, 2016), en una computadora HP LAPTOP- RJSENA2U con Windows 10 y una pantalla de 15,6" para ambos grupos. Además, al grupo de adultos mayores se les administró en primer lugar el test ACE-R (Torralva et. al., 2011), para evaluar las funciones cognitivas $y$ asegurar que superaran el puntaje de corte establecido para su inclusión en este estudio.

\section{Análisis de los datos}

Conforme a lo señalado en la literatura (Oberauer, 2001; Joorman \& Gotlib, 2008) se considerarán como criterios de validez interna que (a) el TR medio en el reconocimiento de los PI debe ser superior al TR medio en el reconocimiento de los $\mathrm{PN}$, debido a que los primeros generan una mayor interferencia, y (b) el porcentaje de respuestas correctas debe ser superior en el reconocimiento de los PN respecto al reconocimiento de los PI. Por otra parte, se espera que el grupo de adultos mayores presente una menor eficiencia inhibitoria que el grupo de jóvenes, lo cual se podrá observar en tanto en mayores TRs como en un menor porcentaje de respuestas correctas para todos los tipos de probes. Para realizar los análisis estadísticos se utilizó el paquete SPSS 23.0 de IBM (2014). Para calcular los TR medios se eliminaron los outliers, los TR individuales que estuvieran a más de $3 \mathrm{DE}$ de la media del sujeto.

Se exploró la distribución normal de las variables en cada grupo a través de la prueba de Kolmogorov-Smirnov. En los adultos jóvenes todas las variables (TR en PI y en PN; precisión en PN y en PI; diferencias en TR entre PI y PN y diferencias en precisión entre $\mathrm{PN}$ y $\mathrm{PI}$ ) tienen un valor $p$ $<.05$ por lo que se asume que su distribución no es normal. En el caso del grupo de adultos mayores, las variables de precisión (tanto en PN como en PI), y de diferencias en precisión entre PN y PI tienen un valor $p<.05$, no siendo normal su distribución; pero para las variables correspondientes a los TR (tanto en PN como en PI y las diferencias entre ellos) sí se puede asumir una distribución normal ya que ambas tienen un valor $\mathrm{p}>.05$. Por lo tanto, se utilizaron pruebas no paramétricas (prueba de suma de rangos de Wilcoxon) para el análisis de la mayoría de las variables salvo para las referidas a los TR en el grupo de adultos mayores, en el que se utilizó una prueba $t$ para muestras relacionadas. Para comparar el rendimiento entre el grupo de adultos mayores respecto al de jóvenes en (a) el TR medio en PI - TR medio en PN y (b) el porcentaje de respuestas correctas en PN - respuestas correctas en PI, se utilizó el Test de U de Mann Whitney para muestras independientes.

\section{Resultados}

En primer lugar se realizó el análisis descriptivo de los principales índices de la tarea de borrado, que se muestran en la Tabla 1. 
Tabla 1

Porcentaje de respuestas correctas y TR medios en PN y PI para los grupos de jóvenes y mayores.

\begin{tabular}{lcccc}
\hline & Grupo & Media & Desviación estándar & Mediana \\
\hline TR en PI & Jóvenes & 1820.64 & 833.66 & 1569.93 \\
\multirow{2}{*}{ TR en PN } & Mayores & 3950.93 & 1901.28 & 3871.60 \\
& Jóvenes & 1454.70 & 728.76 & 1150.51 \\
Precisión en PN & Mayores & 3022.34 & 1361.63 & 2946.17 \\
& Jóvenes & 96.84 & 7.88 & 100.00 \\
Precisión en PI & Mayores & 82.43 & 18.60 & 83.00 \\
& Jóvenes & 81.72 & 18.12 & 83.00 \\
Diferencia TR PI- PN & Mayores & 68.24 & 21.67 & 67.00 \\
& Jóvenes & 365.94 & 428.20 & 219.00 \\
Diferencia precisión & Mayores & 928.68 & 736.25 & 737.40 \\
PN- PI & Jóvenes & 15.13 & 16.57 & 17.00 \\
& Mayores & 14.19 & 18.43 & 11.43 \\
\hline
\end{tabular}

En la Tabla 1 se puede observar que dentro de cada grupo etario el porcentaje de respuestas correctas (precisión) fue superior en PN respecto de PI. Asimismo, el TR en PI fue superior respecto de la condición PN dentro de cada grupo etario.

A fin de comprobar si las diferencias son estadísticamente significativas y si se cumplió con los criterios de validez interna de la tarea, se efectuaron pruebas $t$ y pruebas de suma de rangos de Wilcoxon para muestras relacionadas según la distribución de los datos fuera normal o no. Para ambos grupos, se pudo establecer que las diferencias eran significativas según los criterios de validez interna establecidos y mencionados al inicio del apartado anterior (grupo jóvenes: diferencias en TR PN-PI: $z=4.93 p<.001$ diferencias porcentaje de RC PN-PI: $z=3.89, p<.001$; grupo mayores: diferencias TR PN-PI: $t=5.77, g l: 20$, $p<.001$; diferencias porcentaje de RC PN-PI: $z=$ $2.82, p<.005)$.
Verificación del supuesto de validez a través de cambios maduracionales en el desarrollo

Como ya se mencionó, se espera que los adultos mayores presenten una menor eficiencia inhibitoria que los jóvenes. Esto se reflejaría en el hallazgo de una disminución significativa en el grupo de adultos mayores respecto al de jóvenes en (a) el TR medio en PI - TR medio en PN y (b) el porcentaje de respuestas correctas en PN respuestas correctas en PI. Para contrastar dicho supuesto se utilizó el Test de U de Mann Whitney para muestras independientes.

El análisis de las diferencias en las distribuciones en precisión en PN y en PI entre los dos grupos indicó que estas se deben a circunstancias ajenas a los cambios maduracionales $(z=-0.693 p>$ $.05)$. Por otro lado, el análisis de las diferencias entre ambos grupos para TR en PI y en PN indicó que éstas no se deben a factores externos a las variables estudiadas $(z=-2.85 ; p<.05)$, siendo mayores los tiempos en el grupo de adultos mayores que en el de los jóvenes. 


\section{Discusión}

El objetivo de este trabajo fue presentar evidencia sobre la validez de constructo de una prueba informatizada desarrollada según el modelo de Oberauer (2001), para evaluar la inhibición de borrado en adultos. La inhibición de borrado permite suprimir la información irrelevante que se mantiene activada en el foco atencional y que interfiere con el objetivo de la tarea en curso, incrementando así la eficacia del procesamiento. La teoría postula que los ítems que se vuelven irrelevantes en la fase de señal de la tarea y deben ser borrados generan interferencia en el reconocimiento de los ítems relevantes en la fase de prueba de la tarea, lo cual se evidencia en mayores TR y en un menor porcentaje de respuestas correctas en los ítems irrelevantes que ante ítems nuevos. Los resultados obtenidos se corresponden con dicha explicación, lo cual indica que la tarea de borrado visual constituye un procedimiento válido para la medición de la inhibición de borrado en adultos.

Con respecto al criterio de validez por cambios maduracionales o en el desarrollo, se observó que las diferencias en los TR entre las condiciones PI y PN son estadísticamente significativas entre el grupo de los adultos jóvenes y el grupo de adultos mayores, en ambas condiciones los jóvenes obtuvieron menores TR. Estos resultados indicarían que los adultos mayores tienen una mayor dificultad para borrar la información irrelevante de la memoria de trabajo, lo que se corresponde con las predicciones de la Teoría de la Ineficiencia Inhibitoria de Hasher y Zacks (1988), que sostiene que los adultos mayores en general presentan una menor eficacia en la inhibición activa de la información que los adultos jóvenes. No obstante, de manera coherente o compatible con otros estudios, los resultados obtenidos mostraron una ausencia de diferencias significativas para el índice basado en los porcentajes de respuestas correctas (Joorman \& Gotlib, 2008; Oberauer, 2001), lo que indicaría que la precisión no es un indicador adecuado para la estimación de cambios cognitivos asociados con el envejecimiento.

Por otra parte, cabe destacar que en nuestro trabajo las tasas de respuesta correctas fueron sensiblemente inferiores a las reportadas en otros estudios (Loosli, Rahm, Unterrainer, Weiller, \& Kaller, 2014; Joorman \& Gotlib, 2008) posiblemente debido al carácter abstracto de los estímulos utilizados, pero los resultados presentaron, sin embargo, el mismo sentido que los estudios previos mencionados. Esta afirmación se ve respaldada por el hecho de que tanto en el grupo de adultos mayores como en el de jóvenes la tarea de inhibición de borrado mostró un adecuado ajuste a los criterios de validez interna.

En síntesis, el estudio aporta evidencia empírica de validez en relación con la tarea de borrado visual. Sin embargo, es pertinente señalar algunas limitaciones. En primer lugar, la muestra participante fue seleccionada según disponibilidad y su tamaño es pequeño. Serán necesarios posteriores estudios que incrementen el tamaño de la muestra y la representatividad de la misma a través de su selección aleatoria.

Asimismo, el contraste de otras formas de validez tales como la convergente y la divergente, la predictiva y la capacidad de la misma para detectar cambios maduracionales en otros grupos etarios contribuirán a una validación más amplia de la tarea.

La teoría predice que la inhibición de borrado experimenta una mejoría desde la infancia hasta la adultez (Davidson, Amso, Anderson, \& Diamond, 2006; Introzzi, Canet Juric et. al, 2016), por lo que sería pertinente contrastar si la tarea desarrollada es válida para su aplicación en niños y adolescentes. Otros integrantes del mismo grupo de investigación han realizado estudios para validar esta y otras pruebas de la batería informa- 
tizada TAC en poblaciones infantiles, y de adultos jóvenes (Introzzi, Andrés, et al., 2016; Richard's, Introzzi, Zamora \& Vernucci, 2017)

Tal como fue mencionado en la introducción, la inhibición constituye un proceso cognitivo clave para la adaptación de los sujetos al ambiente. Si bien la literatura actual distingue diferentes tipos inhibitorios, en Argentina no habían sido desarrolladas hasta el momento tareas que permitan la medición de la inhibición de borrado. El disponer de instrumentos válidos para la medición de los diferentes tipos inhibitorios permite una mejor comprensión de la relación de estos procesos con otros procesos cognitivos, así como con variables académicas y la semiología de diferentes trastornos mentales. Con este estudio aspiramos a contribuir a tal meta al presentar una tarea con evidencias de validez para la medición de uno de los componentes de la inhibición, la de borrado.

\section{Referencias}

American Psychological Association. (2017). Ethical principles of psychologists and code of conduct. Recuperado de http://www.apa.org/ethics/code/ethics-code-2017.pdf

Aslan, A., \& Baüml, K. H. T. (2012). Retrieval-induced forgetting in old and very old age. Psychology and Aging, 27(4), 1027-1032. doi: 10.1037/a0028379

Asociación Médica Mundial (2013). Declaración de Helsinki. Principios éticos para las investigaciones con seres humanos. Recuperado de https://www. wma.net/es/policies-post/declaracion-de-helsinki-de-la-amm-principios-eticos-para-las-investigaciones-medicas-en-seres-humanos

Baddeley, A. (2012). Working memory: Theories, models and controversies. Annual Review of Psychology, 63(1), 1-29. doi: 10.1146/annurev-psych-120710-100422

Cohen, J. D., Dunbar, K., \& McClelland, J. L. (1990). On the control of automatic processes: A parallel distributed account of the Stroop effect. Psychological Review, 97(3), 332-361. doi: 10.1037/0033-295X.97.3.332

Cowan, N., Elliot, E. M., Scott-Saults, J. S., Morey, C., Mattox, S., Hismajatullina, A., \& Conway, A. (2005). On the capacity of attention: Its estimation and role in working memory and cognitive aptitudes. Cognitive Psychology, 51(1), 42-100. doi: 10.1016/j. cogpsych.2004.12.001

Davidson, M. C., Amso, D., Anderson, L. C., \& Diamond, A. (2006). Development of cognitive control and executive functions from 4 to 13 years: Evidence from manipulations of memory, inhibition, and task switching. Neuropsychologia, 44(11), 2037-2078. doi: 10.1016/j.neuropsychologia.2006.02.006

Diamond, A. (2013). Executive functions. Annual Review of Psychology, 64(1), 135-168. doi: 10.1146/ annurev-psych-113011-143750

Hasher, L., \& Zacks, R. T. (1988). Working memory, comprehension, and aging: A review and a new view. The Psychology of Learning and Motivation, 22, 193225. doi: 10.1016/s0079-7421(08)60041-9

Hasher, L., Zacks, R. T, \& May, C. P. (1999). Inhibitory control, circadian arousal, and age. En D. Gopher \& A. Koriat (Eds.), Attention \& Performance, XVII, Cognitive Regulation of Performance: Interaction of Theory and Application (pp. 653-675). Cambridge, MA: MIT Press. Recuperado de http://www. psych.utoronto.ca/users/hasherlab/abstracts/hasher_ zacks_may99.htm

Healey, M. K., Ngo, K. W. J., \& Hasher, L. (2014). Below-baseline suppression of competitors during interference resolution by younger but not older adults. Psychological Science, 25, 145-151. doi: 10.1177/0956797613501169

Hogan T. R. (2015). Validez. En T. R. Hogan (Ed.), Pruebas psicológicas: Una introducción práctica ( $2^{\mathrm{a}} \mathrm{ed} ., \mathrm{pp}$. 199-258). México, DF: Manual Moderno.

Hughes, C. P., Berg, L., Danzinger, W. L., Coben, L. A., \& Martin, R. L. (1982). A new clinical scale for staging of dementia. The British Journal of Psychiatry, 
140(6), 566-572. doi: 10.1192/bjp.140.6.566

IBM Corp (2015). IBM SPSS Statistics for Windows, versión 23.0 [software de cómputo]. Armonk, N.Y. USA.

Introzzi, I., Andrés, M. L., Canet-Juric, L., \& Richard's, M. M. (2016). The relationship between the rumination style and perceptual, cognitive and behavioral inhibition. Psychology \& Neuroscience, 9(4), 444-456. doi: 10.1037/pne0000068

Introzzi, I. M., Canet-Juric, L., Aydmune, Y., \& Stelzer, F. (2016). Perspectivas teóricas y evidencia empírica sobre la inhibición. Revista Colombiana de Psicología, 25(2), 351-368. doi: 10.15446/rcp.v25n2.52011

Joorman, J., \& Gotlib, I. H. (2008). Updating the contents of working memory in depression: Interference from irrelevant negative material. Journal of Abnormal Psychology, 117(1), 182-92. doi: 10.1037/0021-843X.117.1.182

Ley $\mathrm{N}^{\circ} 25.326$. Protección de datos personales. Dirección Nacional de Protección de datos personales, Ministerio de Justicia, Seguridad y Derechos humanos, 29 de Noviembre de 2001.

Loosli, S. V., Rahm, B., Unterrainer, J. M., Weiller, C., \& Kaller, C. P. (2014). Developmental change in proactive interference across the life span: Evidence from two working memory tasks. developmental Psychology, 50(4), 1060.doi: 10.1037/a0035231

Lustig, C., Hasher, L., \& Zacks, R. T. (2008). Inhibitory deficit theory: Recent development in a "new view". En D. S. Gorfein \& C. M. MacLeod (Eds.), The place of inhibition in cognition (pp. 145-162). Washington, DC: American Psychological Association. doi: 10.1037/11587-008

Mathuranath, P. S., Nestor, P. J., Berrios, G. E., Rakowicz, W., \& Hodges, J. R. (2000). A brief cognitive test battery to differentiate Alzheimer's disease and frontotemporal dementia. Neurology, 55(11), 1613-1620. doi: 10.1212/01.wnl.0000434309.85312.19

Miyake, A., \& Friedman, N. P. (2012). The nature and organization of individual differences in executive functions: Four general conclusions. Current Di- rections in Psychological Science, 21(1), 8-14. doi: 10.1177/0963721411429458

Morton, J. B., \& Munakata, Y. (2002). Active versus latent representations: A neural network model of perseveration, dissociation, and decalage. Developmental Psychobiology, 40(3), 255-265. doi: 10.1002/ dev. 10033

Oberauer, K. (2001). Removing irrelevant information from working memory: A cognitive aging study with the modified Sternberg task. Journal of Experimental Psychology: Learning, Memory and Cognition, 27(4), 948-957. doi: 10.1037/0278-7393.27.4.948

Oberauer, K. (2005). Control of the contents of working memory -A comparison of two paradigms and two age groups. Journal of Experimental Psychology: Learning, Memory and Cognition, 31(4), 714-728. doi: 10.1037/0278-7393.31.4.714

Ortega, A., Gómez-Ariza, C. J., Román, P., \& Bajo, M. T. (2012). Memory inhibition, aging and the executive deficit hypothesis. Journal of Experimental Psychology: Learning, Memory and Cognition, 38(1), 178186. doi: $10.1037 / \mathrm{a} 0024510$

Richard's, M., Introzzi, I., Zamora, E. \& Vernucci, S. (2017). Analysis of Internal and External Validity Criteria for a Computerized Visual Search Task. A pilot study. Applied Neuropsychology: Child, 6(2) 110-119 (print), 2162-2973 (Online). doi: 10.1080/21622965.2015.1083433

Sahakyan, L., Delaney, P. F., \& Waldum, E. R. (2008). Intentional forgetting is easier after two" shots" than one. Journal of Experimental Psychology: Learning, Memory, and Cognition, 34(2), 408. doi: 10.1037/0278-7393.34.2.408

Sego, S. A., Golding, J. M., \& Gottlob, L. R. (2006). Directed forgetting in older adults using the item and list methods. Aging, Neuropsychology, and Cognition, 13, 95-114. doi: 10.1080/138255890968682

Sternberg, S. (1969). Memory scanning: Mental processes revealed by reaction-time experiments. American Scientist, 57, 421-457. Recuperado de http://www. psych.upenn.edu/ saul 
Torralva, T., Roca, M., Gleichgerrcht, E., Bonifacio, A., Raimondi, C., \& Manes F. (2011). Validación de la versión en español del Addenbrooke's Cognitive Examination-Revisado (ACE-R). Neurología, 26(6), 351-356. doi: 10.1016/j.nrl.2010.10.013

Vadaga, K. K., Blair, M., \& Li, K. Z. H. (2015). Are age-related differences uniform across different inhibitory functions? Journal of Gerontology: Serie B: Psychological Sciences and Social Sciences, 71(4), 641649. doi: 10.1093/geronb/gbv002

Vivas, J., Vivas, L., Comesaña, A., Coni, A. G., \& Vorano, A. (2016). Spanish semantic feature production norms for 400 concrete concepts. Behavior Research Methods, 49(3), 1095-1106. doi: 10.3758/ s13428-016-0777-2

Yao, Z., Wu, J., Zhang, Y., \& Wang, Z. (2016). Norms of valence, arousal, concreteness, familiarity, imageability, and context availability for 1100 chinese words. Behavior Research Methods, 49(4), 1374-1385. doi: 10.3758/s13428-016-0793-2 